\title{
Efecto del agua aplicada en las relaciones hídricas y productividad de la vid 'Crimson Seedless'
}

\author{
Raúl Ferreyra( ${ }^{(1)}$, Gabriel Selles $^{(1)}$, Hermán Silva ${ }^{(2)}$, Rodrigo Ahumada ${ }^{(1)}$, Iván Muñoz ${ }^{(1)}$ y Verónica Muñoz(2)
}

\begin{abstract}
(1)Instituto de Investigaciones Agropecuarias, Centro Regional de Investigaciones La Platina, Casilla 439, Correo 3, Código Postal 7083150, Santiago, Chile. E-mail: rferreyr@inia.cl, gselles@inia.cl, rahumada@riegoaconcagua.cl, imunoz@inia.cl (2)Universidad de Chile, Facultad de Ciencias Agronómicas, Santa Rosa 11315, Santiago, Chile. E-mail: hsilva@uchile.cl, veroagr@hotmail.com
\end{abstract}

Resumen - Este estudio fue dirigido para evaluar la relación agua-rendimiento en vid de mesa cv. Crimson y establecer valores críticos para las mediciones del estado hídrico de las plantas. Los estudios de campo se desarrollaron durante tres años, en el Valle de Aconcagua, Chile, a $32^{\circ} 47^{\prime} \mathrm{S}$ y $70^{\circ} 42^{\prime} \mathrm{O}$, en un suelo de textura franco arcillosa. Se proporcionaron a las plantas diferentes cantidades de agua de riego entre 40 y $100 \%$ de la evapotranspiración del cultivo (Etc). El potencial hídrico xilemático medido a mediodía ( $\Psi x m i n)$ y la conductancia estomática estuvieron estrechamente relacionados con el déficit de agua impuesto y el rendimiento obtenido. Los rendimientos de la vid disminuyeron respecto al agua aplicada en el rango de los tratamientos estudiados. Sesenta por ciento de restricción de la Etc redujo 22\% del rendimiento. Cuando la planta mantuvo $\Psi x m i n$ mayor que -0,75 MPa entre cuaja y pinta, la producción y los calibres fueron mayores.

Términos para indexación: potencial hídrico xilemático, conductancia estomática, uva de mesa.

\section{Effect of applied water on water relations and productivity of 'Crimson Seedless' table grapes}

\begin{abstract}
This study aimed to evaluate the relationship between water and production in 'Crimson Seedless' table grapes, and to establish threshold values for plants water status. Field experiments were carried out, during a three-year period, in the Aconcagua Valley, Chile, at $32^{\circ} 47^{\prime} \mathrm{S}$ and $70^{\circ} 42^{\prime} \mathrm{W}$, in a clay-loamy textured soil. Different irrigation water amounts were applied, between 40 and 100\% crop evapotranspiration (Etc). Stem water potential measured at midday ( $\Psi$ xmin) and stomatal conductance were closely related to water shortage and yield obtained. Table grape yields decreased in comparison with applied water within the range of studied treatments. Sixty per cent Etc restriction decreased yields in 22\%. When plants maintained $\Psi$ xmin greater than $-0.75 \mathrm{MPa}$, between berry set and veraison, yield and berry size were high.
\end{abstract}

Index terms: stem water potential, stomatal conductance, table grapes.

\section{Introducción}

Las vides se desarrollan en zonas templadas, pero son cultivadas en todas las regiones climáticas, excepto las de inviernos extremadamente fríos; por eso, en muchos casos, son plantadas en condiciones ambientales desfavorables, donde el suministro de agua y los suelos presentan restricciones para su desarrollo, lo que limita la productividad y calidad de la fruta. En la zona central de Chile (V Región), donde se encuentra el 22,8\% de las plantaciones de vides de mesa, la mayoría de los suelos cultivados con esta especie presentan baja macroporosidad, comparada con los valores señalados por Richards (1983) como limitantes para su desarrollo.

La producción media en vid de mesa, dependiendo de la variedad, en la zona central de Chile, varia de 14,8 a 16,4 Mg ha-1 exportables. Sin embargo, el potencial productivo, en condiciones ambientales favorables, permite alcanzar 24,6 $\mathrm{Mg} \mathrm{ha}^{-1}$, como lo muestran los rendimientos de algunos agricultores de la zona señalada.

Uno de los principales factores que afectan la producción y calidad de la fruta, sobre todo en condiciones de suelo desfavorables para el desarrollo de la vid, es una inadecuada relación entre el agua y el aire en el suelo, producto del manejo del riego, que puede inducir déficit hídrico o exceso de agua en el suelo.

La falta de agua, durante el crecimiento de la vid de mesa, limita la producción y la calidad de la fruta, particularmente si el déficit hídrico se produce entre floración y pinta, por lo que es importante detectar, en este período, cualquier déficit hídrico, incluso moderado, lo más temprano posible. 
El exceso de agua en el suelo disminuye la disponibilidad de oxígeno para las raíces. Un déficit de oxígeno inhibe la fotosíntesis, el transporte de hidratos de carbono, y causa cierre de estomas y un menor crecimiento de las raíces (Kozlowski, 1997). Una estrategia de manejo del riego para optimizar la relación agua-aire en el suelo requiere información respecto a las pérdidas de rendimiento asociada a déficit de agua, al impacto del estrés hídrico en la respuesta fisiológica de la vid y a técnicas para evaluar si el régimen de riego es adecuado.

Mediciones periódicas del estado hídrico del suelo, ya sea del potencial mátrico $(\Psi \mathrm{m})$ o del contenido de humedad de este, se han utilizado normalmente para establecer niveles de déficit de agua y necesidades de riego de las plantas. Diferentes estudios han demostrado que el potencial hídrico xilemático ( $\Psi x)$, medido con cámara de presión, es un buen indicador del estrés hídrico en árboles frutales. Naor et al. (1995) encontraran una buena correlación entre $\Psi \mathrm{x}$ y el rendimiento en manzanos. Datos similares encontraran Ferreyra et al. (2002, 2004) en duraznero y vides viníferas. Naor (1998) y Ferreyra et al. (2002) comprobaron que la conductancia estomática en nectarines y vid vinífera está altamente correlacionada con $\Psi x$. Por lo cual, el potencial hídrico xilemático ( $\Psi$ xmin), medido a mediodía, ha sido propuesto por varios autores como un indicador estándar para determinar el estado hídrico de la planta, con fines de riego (Fereres \& Goldhamer, 1990; Shackel et al., 1997; Naor, 2001; Ferreyra et al., 2002).

Aunque un numero importante de literatura caracterize el impacto del déficit de agua en respuesta fisiológica de la vid, hay poca información que cuantitativamente relacione la producción con la cantidad de agua de riego aplicada.

Este estudio fue dirigido para evaluar la relación aguarendimiento para la vid de mesa 'Crimson Seedless' y establecer valores críticos para las mediciones del estado hídrico de las plantas, de manera a disponer de información que permita manejar el riego optimizándose la relación agua-aire en el suelo.

\section{Material y Métodos}

El ensayo se llevó a cabo durante tres temporadas (2002/2003, 2003/2004 y 2004/2005), en vid de mesa variedad 'Crimson Seedless', de siete años de edad, conducida en parronal español. El ensayo se ubicó en el Valle de Aconcagua, comuna de Curimón, Chile, a $32^{\circ} 47^{\prime} \mathrm{S}$ y $\left.70^{\circ} 42^{\prime} \mathrm{O}\right)$. El suelo del sector de estudio corresponde a la serie Curimón (Fluventic Haploxerol), de textura franco arcillosa de 1,5 m de profundidad, con una densidad aparente promedio de 1,44 $\mathrm{g} \mathrm{cm}^{-3}$. El área de ensayo ocupó una superficie de $4.032 \mathrm{~m}^{2}$, con 384 plantas en total, con un diseño experimental de bloques al azar, con cuatro tratamientos y cuatro repeticiones.

Se definieron cuatro tratamientos de riego, durante toda la temporada: $\mathrm{T} 1$, riego a $100 \%$ de la evapotranspiración del cultivo (Etc); T2, riego con 75\% de la Etc; T3, riego con 50\% de la Etc; y T4, un régimen variable de riego entre 0 y $100 \%$ de la Etc. En las dos primeras temporadas (2002/2003, 2003/2004), T4 no fue regado entre el inicio de brotación y 73 días después de la brotación (ddb), y entre 83 y $113 \mathrm{ddb}$; el resto de la temporada se regó igual que T1. Durante la última temporada, T4 fue regado igual que T1, salvo en el período comprendido entre 65 y $101 \mathrm{ddb}$, y entre 124 y 152 ddb, en que no fue regado. En promedio, T4 recibió entre 50 y 58\% del agua aplicada a T1.

Los requerimientos hídricos de las plantas se estimaron a partir de la evapotranspiración de referencia (Eto, método de Penman-Montheith), obtenida de una estación meteorológica automática ubicada en el predio. Durante la primera temporada, para determinar la Etc se emplearon los coeficientes de cultivos, Kc, que utilizan los agricultores del Valle de Aconcagua. A partir de la segunda temporada, los Kc se determinaron de acuerdo al porcentaje de sombramiento del parrón, según la metodología de Williams et al. (2003). El régimen de riego fue de baja frecuencia, con riegos cada dos a cuatro días, dependientes de la Eto, como lo recomiendan Selles et al. (2003) para suelos de textura finas del Valle de Aconcagua.

El sistema de riego fue por goteo, constituido por dos líneas de riego por hilera de plantas, separadas a 0,9 m entre ellas. Durante las dos primeras temporadas, las líneas permanecieron fijas en el terreno. Durante la última temporada, cada tres riegos, las líneas de goteo fueron desplazadas hacia el centro de la entre hilera, para ampliar el bulbo de mojamiento.

La variación de humedad del suelo, en cada tratamiento de riego, se midió utilizándose una sonda capacitiva (Delta-T probe model PR1). En una repetición por tratamiento (en la parte central del ensayo), se instalaron cinco tubos de acceso, perpendiculares a la línea de plantación, cada 0,3 m desde la hilera de plantas hacia el centro de la entre hilera, hasta 1,2 m de 
distancia. El contenido de humedad del suelo se midió a $0,10,0,20,0,40,0,60$ y $1 \mathrm{~m}$ de profundidad. Las mediciones se realizaron semanalmente. Para relacionar los contenidos de humedad, medidos con la humedad aprovechable del suelo (HA), durante el invierno de 2003, se midió el contenido de humedad a capacidad de campo (CC), in situ, de acuerdo a la metodología propuesta por Cassel \& Nielsen (1986). El contenido de agua a CC se midió con la sonda capacitiva, obteniéndose un valor de $344 \mathrm{~mm}$ para el perfil de suelo.

El potencial hídrico de las plantas se midió utilizándose el método de la cámara de presión (Scholander et al., 1965). Las mediciones se realizaron tanto en hojas expuestas al sol (potencial de hoja, $\Psi$ f) como en hojas sombreadas, las cuales fueron cubiertas con una bolsa de material de aluminio (Meyer \& Reicosky, 1985; Schakel et al., 1997), con el objeto de determinar el potencial hídrico xilemático ( $\Psi \mathrm{x})$. Estas mediciones se realizaron a mediodía, cuando el $\Psi$ y y el $\Psi$ x alcanzan su valor mínimo ( $\Psi$ fmin y el $\Psi x m i n)$, con una frecuencia de dos veces por semana, en dos plantas de cada tratamiento. Adicionalmente, cada 15 días se midió el $\Psi$ xmin en todas las repeticiones de cada tratamiento. Ocasionalmente, se realizaron mediciones diarias del potencial hídrico de las plantas, desde antes de la salida del sol (potencial hídrico de base, $\Psi$ b), hasta el anochecer.

La conductancia estomática de las hojas (gs) se determinó, utilizándose un porómetro de equilibrio Li-Cor modelo LI-1600. Las mediciones se realizaron en seis hojas de dos plantas por tratamiento, con una frecuencia semanal, a las 9 y $14 \mathrm{~h}$.

El porcentaje de sombreamiento se estimó a partir de la radiación solar fotosintéticamente activa (PAR), interceptada por el follaje de la planta a mediodía (Suckel, 2001). Las mediciones se realizaron semanalmente, en un cuadrante constituido por seis plantas centrales de cada tratamiento, utilizándose un radiómetro de barra ACCUPAR (Decagón).
Durante el proceso de desarrollo de las bayas, en 72 bayas por tratamiento, se midió semanalmente su diámetro, utilizándose un micrómetro manual, entre cuaja e inicio de cosecha, a fin de establecer las curvas de crecimiento de las bayas en cada tratamiento. La producción de fruta se evaluó en las plantas centrales de cada repetición.

A los resultados obtenidos se les aplicó un análisis de varianza (ANDEVA), y el test de Duncan $(p<0,05)$, cuando se encontraron diferencias significativas.

\section{Resultados y Discusión}

Los volúmenes reales de agua, aplicados en cada tratamiento, se presentan en el Cuadro 1.

Los tratamientos de riego produjeron diferencias sustanciales en el contenido de humedad del suelo, a medida que la estación de crecimiento avanzaba (Figura 1). En las temporadas 2002/2003, 2003/2004 y $2004 / 2005$, las lluvias durante el invierno elevaron el contenido de humedad del suelo a niveles cercanos a la capacidad de campo, antes que los tratamientos de riego comenzaran. Por eso, al inicio de cada estación de crecimiento, los contenidos de humedad del suelo fueron similares en todos los tratamientos. Los contenidos de humedad del suelo se expresaron en milímetros hasta $0,6 \mathrm{~m}$ de profundidad, ya que las mediciones realizadas a $1 \mathrm{~m}$ de profundidad presentaron muy poca variación, en relación a las otras estratas de suelo, lo que indica una baja extracción de agua a este nivel.

Como era de esperarse, solamente el tratamiento $100 \%$ de la Etc mantuvo el suelo con un alto contenido de humedad, a lo largo de toda la estación de crecimiento. La evolución del contenido de humedad de suelo (Hs), en la temporada 2003/2004, se indica en la Figura 1. En T1 el Hs varió entre 81 y 96\% de CC, T2 varió entre 70 y $80 \%$ de CC, T3 entre 59 y 83\% de CC. Finalmente, T4, al 79 ddb, registró el valor mínimo de agua en el

Cuadro 1. Evapotranspiración y cantidad neta de agua aplicada en cada tratamiento de riego, durante las temporadas 2002/2003, 2003/2004 y 2004/2005(1).

\begin{tabular}{|c|c|c|c|c|c|c|c|}
\hline \multirow[t]{2}{*}{ Año } & \multirow[t]{2}{*}{$\mathrm{Pp}(\mathrm{mm})$} & \multirow[t]{2}{*}{ Eto $(\mathrm{mm})$} & \multirow[t]{2}{*}{ Etc $(\mathrm{mm})$} & \multicolumn{4}{|c|}{ Agua aplicada (mm) } \\
\hline & & & & $\mathrm{T} 1$ & $\mathrm{~T} 2$ & $\mathrm{~T} 3$ & $\mathrm{~T} 4$ \\
\hline $2002 / 2003$ & 446 & 903,8 & 851,7 & 803,9 & 546,7 & 302,8 & 385,5 \\
\hline $2003 / 2004$ & 119 & $1.061,0$ & $1.127,4$ & $1.056,4$ & 759,2 & 418,9 & 708,8 \\
\hline $2004 / 2005$ & 160 & 876,2 & 897,8 & 919,3 & 614,3 & 409,2 & 582,2 \\
\hline Promedio & 242 & 947,0 & 959,0 & 926,5 & 640,0 & 376,9 & 558,9 \\
\hline Etc $(\%)$ & & & 100,00 & 96,66 & 67,74 & 39,31 & 58,28 \\
\hline
\end{tabular}

(1)Eto: evapotranspiración de referencia; Etc: evapotranspiración del cultivo; Pp: precipitación invernal. 
suelo, que fue de $49 \%$ de la CC, y el máximo valor alcanzado fue de $79,4 \%$ de CC. El Hs varió de forma similar durante las tres temporadas.

La variación diurna del $\Psi$ f y del $\Psi x$ de la vid, de los tratamientos T1 y T4, el día 79 ddb (temporada 2003/2004), que corresponde al día de máximo estrés para el tratamiento T4, se ilustra en la Figura 2. El potencial hídrico de la planta es máximo antes del amanecer (potencial hídrico de base, $\Psi$ b); después de la salida del sol, tanto $\Psi \mathrm{f}$ como $\Psi \mathrm{x}$ disminuyen rápidamente, siguiendo estrechamente el incremento del déficit de presión de vapor del aire, DPV (Figura 2), para llegar a un valor mínimo al mediodía, entre las 13 y $16 \mathrm{~h}$ aproximadamente, permaneciendo relativamente estable por las siguientes 3 a 4 horas. Después de esto, al disminuir el DPV, las plantas comienzan a recuperar el $\Psi$ hasta llegar, a las 21h, a un valor similar al medido a inicios del

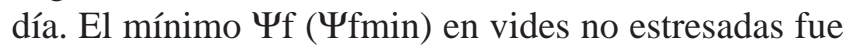
de -0,85 MPa, mientras que en plantas estresadas fue de -1,15 MPa. El mínimo $\Psi x$ ( $\Psi x m i n)$ en plantas no estresadas fue de $-0,77 \mathrm{MPa}$, y en plantas estresadas fue de -1,1 MPa (Figura 2).

A pesar de la diferencia en los potenciales hídricos con que la planta comienza el día (potencial hídrico de base), la conductancia estomática, medida a las $7 \mathrm{~h}$ de la mañana, es similar en ambos tratamientos (1,72 y $1,66 \mathrm{~cm} \mathrm{~s}^{-1}$ para T1 y T4, respectivamente). Diferencias significativas se presentan en solo a las $14 \mathrm{~h} 30\left(0,4 \mathrm{~cm} \mathrm{~s}^{-1} \mathrm{en}\right.$
T1 y $0,08 \mathrm{~cm} \mathrm{~s}^{-1}$ en T4). Con respecto a la evolución diaria de la conductancia estomática, dos puntos son relevantes: los valores máximos se producen temprano en la mañana (7 a 8h), con PAR entre 15 y $60 \mu \mathrm{mol} \mathrm{cm}^{-2} \mathrm{~s}^{-1} \mathrm{y}$ DPV del aires inferior a $1 \mathrm{kPa}$; los estomas se cierran entre las 14 y 15h, momento en que el DPV del aire es máximo, y no se abren posteriormente, aún cuando el DPV del aire disminuye.

Las plantas presentan una fuerte regulación de las pérdidas de agua por transpiración, durante las horas de mayor demanda, lo que puede permitir mantener un potencial hídrico estable en este período, sin que se manifiesten grandes diferencias entre los tratamientos de riego, salvo cuando los déficit son extremos, como es el caso de T4 en la Figura 2.

Una evolución diaria similar del comportamiento estomático se ha encontrado en otras variedades de vides, como en la cultivar Italia (De Lorenzi \& Rana, 2000), y en la cultivar Thompson Seedless (Felmer \& Ortiz, 1997). Valores máximos de conductancia estomática de $0,66 \mathrm{~cm} \mathrm{~s}^{-1}$ han sido medidos por Van Zyl (1984), en vides 'Colombar'; de 0,4 a 0,3 $\mathrm{cm} \mathrm{s}^{-1}$ por Felmer \& Ortiz (1997) en 'Thomson Seedless'; y de $0,4 \mathrm{~cm} \mathrm{~s}^{-1}$ por De Lorenzi \& Rana (2000) para 'Italia'. Los valores máximos medidos en este trabajo son superiores a $1 \mathrm{~cm} \mathrm{~s}^{-1}$, antes de las $8 \mathrm{~h}$ de la mañana; sin embargo valores medios a las $9 \mathrm{~h}$ son muy similares a los reportados por los autores indicados.

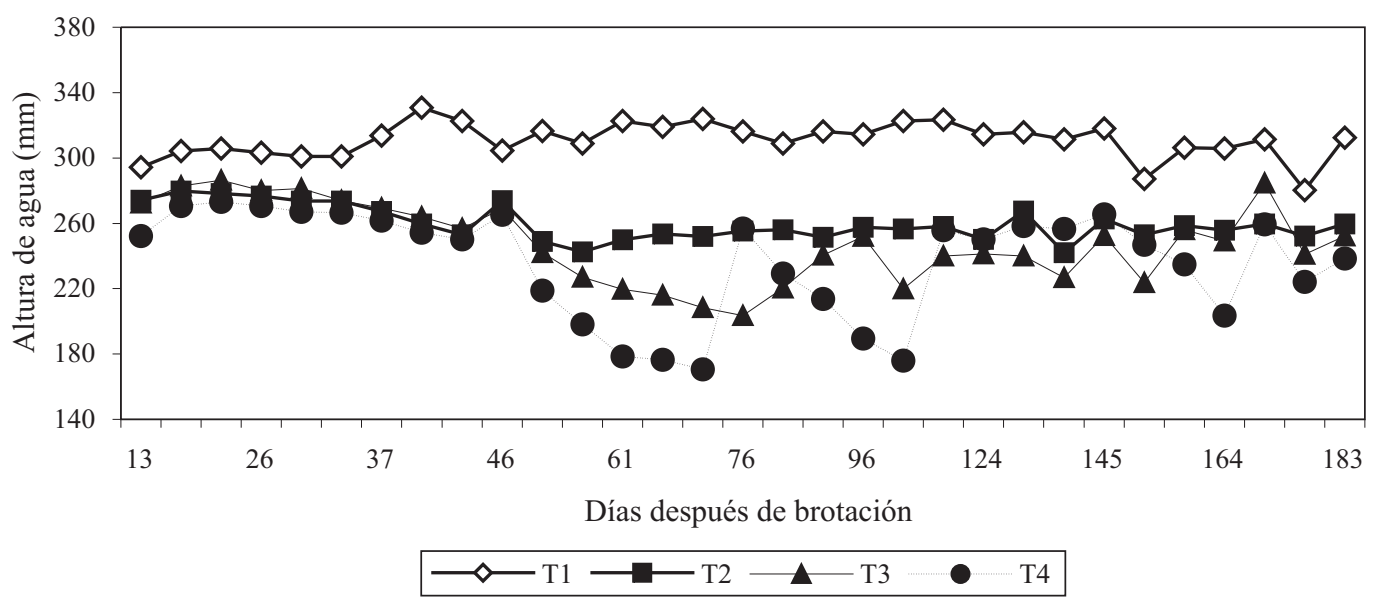

Figura 1. Evolución del contenido de humedad del suelo, en los diferentes tratamientos de riego, expresada en altura de agua por profundidad de suelo, para la temporada 2003/2004. T1: riego a 100\% de la Etc durante toda la temporada; T2: riego con 75\% de la Etc durante toda la temporada; T3: 50\% de la Etc durante toda la temporada; T4: régimen variable de riego entre 0 y 100\% durante toda la temporada; durante las dos primeras temporadas el tratamiento T4 no fue regado desde inicio de brotación hasta el día 4/12 (81 y 78 días después de brotación, respectivamente). 
La Figura 3 muestra la evolución del $\Psi$ xmin para todos los tratamientos de riego, durante la temporada 2003/2004. La evolución de los $\Psi$ xmin fue similar a de las otras temporadas, salvo para el tratamiento $\mathrm{T} 4$, que en el periodo 2004/2005 fue regado similarmente a T1 hasta $65 \mathrm{ddb}$, momento a partir del que se le suspendió el riego hasta $104 \mathrm{ddb}$. Los menores valores se observan con posterioridad a pinta.

En términos generales, el tratamiento T1 presentó los mayores valores de $\Psi$ xmin, situación que se hace más clara, a medida que se restringe la aplicación de agua. Sin embargo, se observan diferencias significativas entre tratamientos solo en algunas fechas de medición. No existieron diferencias significativas entre T1 y T2. Diferencias significativas entre T1 y T3 se presentaron entre los 70 y 93 ddb, y entre 114 y 126 ddb. Entre T1 y
T4, las diferencias significativas existieron entre los 64 y 79, y 98 y 114 ddb.

Los valores más altos de $\Psi$ xmin se midieron temprano en la temporada, $23 \mathrm{ddb}$, y variaron entre -0,42 y -0,55 MPa. Los valores mínimos se observaron hacia el final de la temporada y fueron de -0,98, -1,0, -1,17 y -1,2 MPa, para T1, T2, T3 y T4, respectivamente. Los tratamientos con estrés más severo (T3 y T4) mostraron una mayor disminución de los $\Psi$ xmin.

En términos generales, la evolución presentada por los $\Psi$ xmin, de los diferentes tratamientos, es similar a la observada corrientemente en especies frutales (Ferreyra et al., 2002; Padgett-Jhonson et al., 2003; Intrigliolo \& Castel, 2004), en el sentido que los $\Psi x$ xmin diarios tienden a disminuir a lo largo de la estación de crecimiento. Esto se asocia a la mayor demanda evaporativa de la

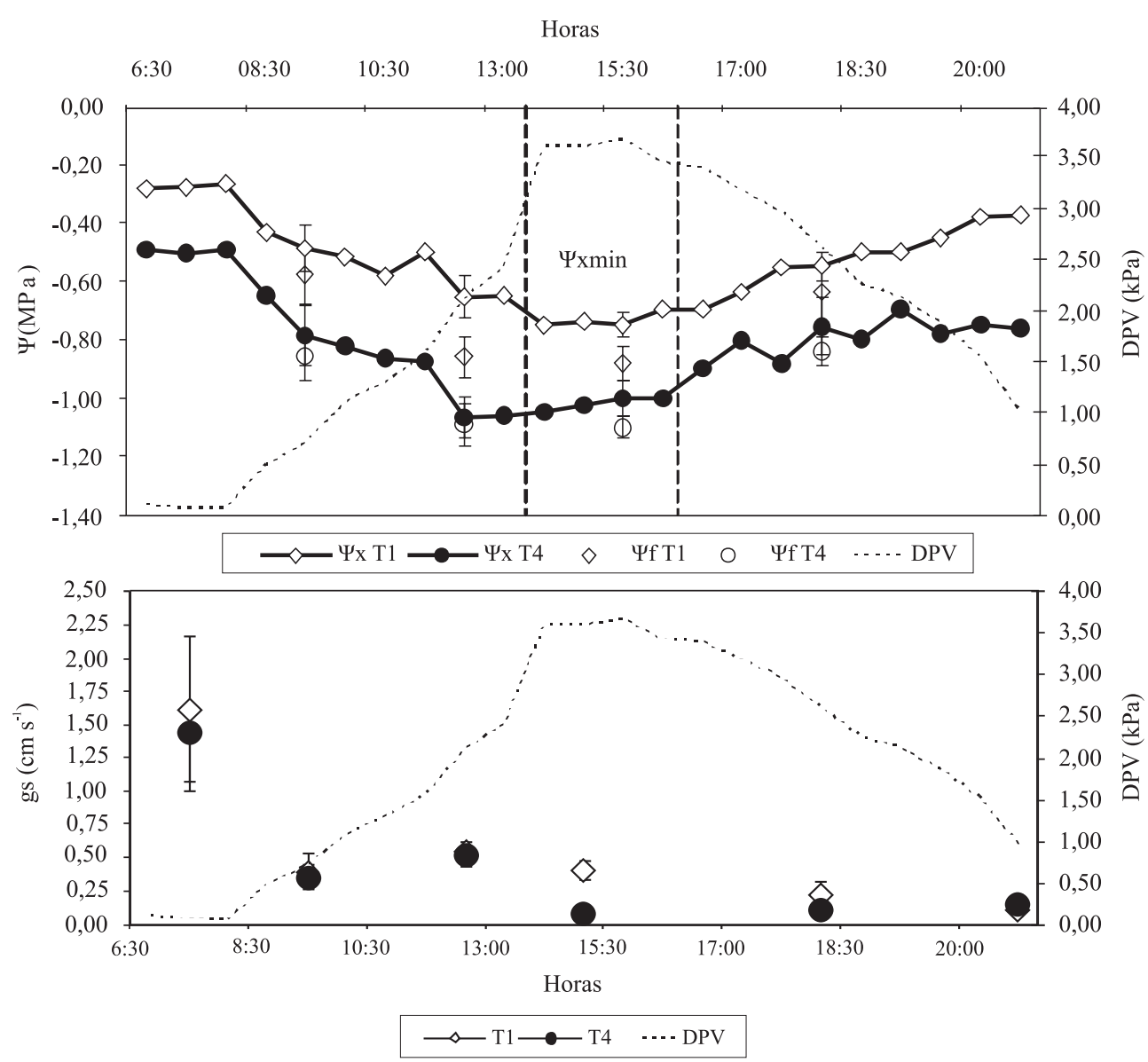

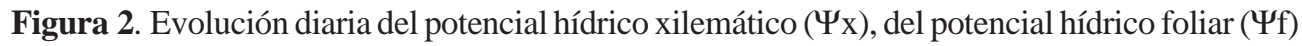
(gráfico superior), y de la conductancia estomática (gs) (gráfico inferior), durante el día 79 ddb, temporada 2003/2004. El contenido de humedad de suelo (Hs) fue de 323 mm para T1 y $171 \mathrm{~mm}$ para T4. A la derecha se presenta el déficit de presión de vapor del aire (DPV). Barras verticales representan error estándar. $\Psi: \mathrm{n}=3$. gs: $\mathrm{n}=6$. 
atmósfera, a la edad de las hojas y a reducción general de la disponibilidad de agua del suelo, a medida que
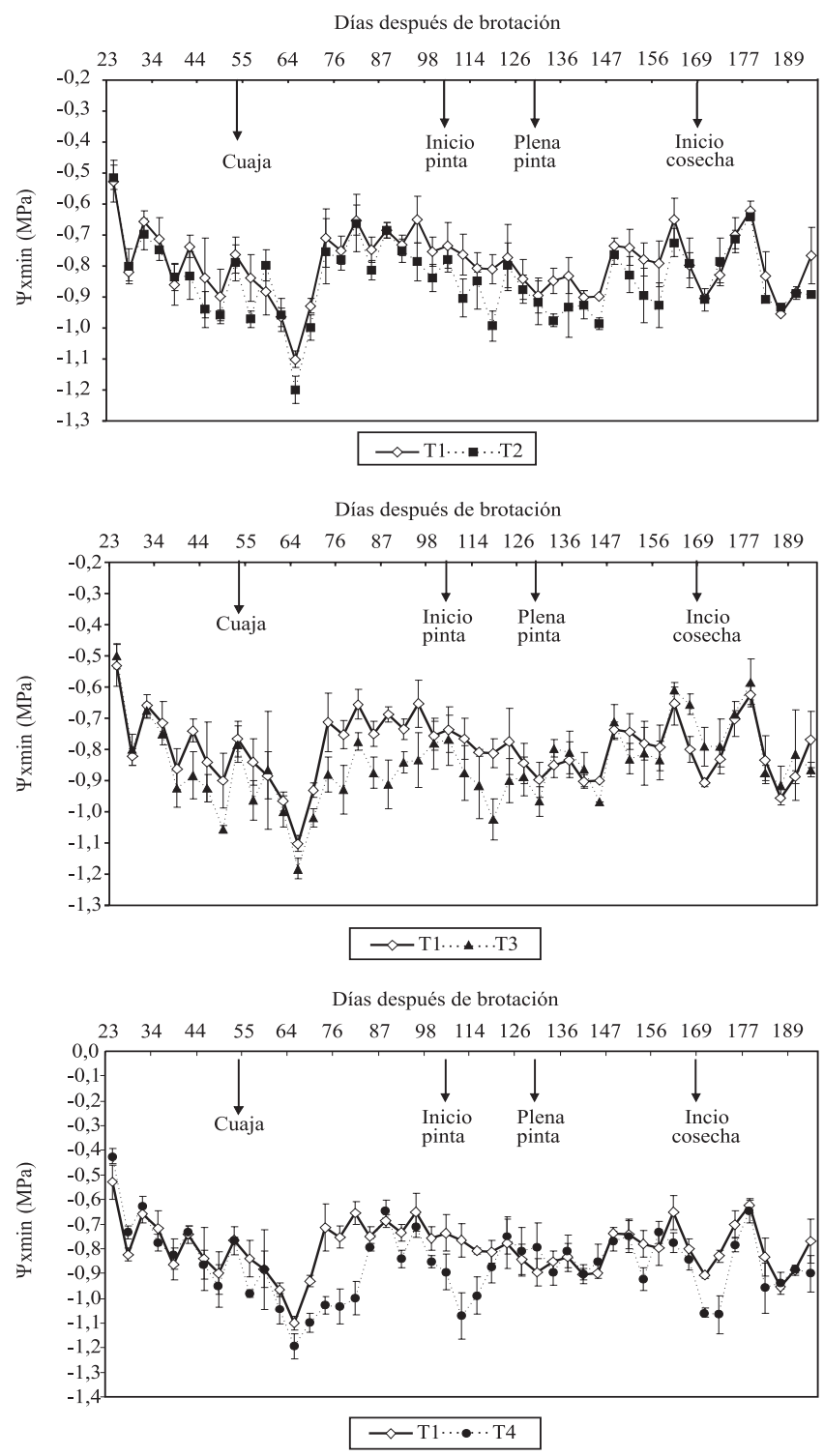

Figura 3. Evolución estacional del potencial xilemático mínimo ( $\Psi x m i n)$, durante la temporada 2003/2004. T1: riego a $100 \%$ de la Etc durante toda la temporada; T2: riego con $75 \%$ de la Etc durante toda la temporada; T3: riego con 50\% de la Etc durante toda la temporada; T4: régimen variable de riego entre 0 y $100 \%$ durante toda la temporada; durante las dos primeras temporadas este tratamiento no fue regado desde inicio de brotación hasta el día 4/12 (81 y 78 días después de brotación, respectivamente); durante la última temporada T4 fue regado igual que T1 hasta 65 días después de brotación, posteriormente fue sometido a restricciones de riego (sin riego y riego a $100 \%$ Etc). Barras verticales representan error estándar. $\Psi: \mathrm{n}=3$. transcurre la temporada. Cuando a T4 se le reanuda el riego, los valores de $\Psi$ xmin vuelven rápidamente a alcanzar valores similares a T1 y T2 (100 y 75\% de Etc) (Figura 3); respuesta parecida ha sido encontrada en otras especies frutales (Intrigliolo \& Castel, 2004). En diferentes especies de vitis, Padgett-Jhonson et al. (2003) indican que para plantas bien regadas los valores de $\Psi$ xmin diario varían entre -0,5 MPa, al inicio de temporada, y -0,9 MPa, 120 días después, a pesar de que el $\Psi \mathrm{x}$ medido antes del amanecer permanece relativamente constante.

En relación con resultados obtenidos, en suelos más delgados, por otros investigadores (Castro, 1997; Felmer \& Ortiz, 1997; Ferreyra et al., 2002; Mateo, 2003), la poca magnitud de diferencia de potenciales hídricos, observados en este ensayo, puede estar relacionada con la profundidad y capacidad de retención del suelo, ya que con el lento desarrollo del déficit hídrico, que se produce en estas condiciones, las plantas pueden adaptarse disminuyendo su área foliar y, por consiguiente, la transpiración, manteniendo potenciales hídricos más o menos estables (Jones, 2004). En este ensayo, las plantas que recibieron menos agua presentaron un menor índice de área foliar (IAF) que las plantas bien regadas (3,7 a 4 en T1 y T2, y 2,5 a 3 em T3 y T4), situación que puede ayudar a explicar la evolución presentada por el $\Psi x m i n$ de los tratamientos T3 y T4.

En vides de variedad 'Thompson Seedeless', cultivadas en suelos delgados, Castro (1997), Felmer y Ortiz (1997) y Mateo (2003) encontraron valores

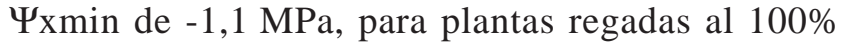
de la Etc, y entre -1,2 a -1,3 MPa, con aplicaciones de agua del 50\% de la Etc. Sin embargo, en plantas de 'Thompson Seedless', cultivadas en suelos profundos del Valle de Aconcagua, regadas al 100\% de la Etc, los $\Psi$ xmin fluctuaron entre -0,6 y -0,7 MPa (Selles et al 2003).

En vides 'Cabernet Sauvignon', plantadas en suelos delgados aluviales, Ferreyra et al. (2002) midieron $\Psi$ xmin de $-1 \mathrm{MPa}$, en plantas regadas al $100 \%$ de la Etc, y entre $-1,3$ y -1,4 MPa con aplicaciones de agua del 50\% de Etc. Habu-Hilal et al. (2000) determinaron, también en 'Cabernet Sauvignon', que plantas regadas con el 75\% de la Etc presentan $\Psi$ xmin 0,05 MPa más negativos que plantas regadas al $100 \%$ de la Etc; y con riego a $50 \%$ de la Etc, la diferencia fue de 0,14 MPa. Aún en 'Cabernet Sauvignon', Chone et al (2001) encontraron, en plantas sin riego, que el $\Psi$ xmin llegaba a -1,4 MPa, sin embargo, la magnitud dependía de la profundidad de 
suelo y de la exploración del sistema radicular; según estos autores, en suelos más delgados, el $\Psi$ xmin de plantas no regadas era más negativo, del orden de 0,3 MPa, que lo que presentaban plantas, también sin riego, en suelos más profundos, de la misma textura.

En este ensayo, en ninguna de las tres temporadas los tratamientos de riego T3 y T4 presentaron valores de $\Psi$ xmin inferiores a -1,2 MPa. Los contenidos de humedad mínimos en T3 fueron, en promedio, de 65\% de capacidad de campo (CC) y, en T4, esta disminuyó hasta valores de $50 \%$ de CC al final de los ciclos de restricción hídrica.

En este trabajo, se pudo observar que el estado hídrico de la planta está estrechamente correlacionado a la tasa de crecimiento de la baya, entre cuaja y pinta (fases I y II) (Figura 4), y al rendimiento relativo (Figura 5), de lo cual se desprende que el $\Psi$ xmin, medido con la cámara de presión, puede ser una buena herramienta para el control de los programas de riego; sin embargo la magnitud de la diferencia de los $\Psi$ xmin entre distintos régimenes de riego dependerá de la capacidad de almacenamiento y profundidad del suelo (Chone et al., 2001)

La producción exportable, obtenida en los diferentes tratamientos, varió entre $25 \mathrm{Mg} \mathrm{ha}^{-1}$, en T1, y 8,8 $\mathrm{Mg} \mathrm{ha}^{-1}$ en T4 (Cuadro 2). En el tratamiento T1, donde se aplicó en promedio 97,8\% de la Etc, se obtuvo un rendimiento

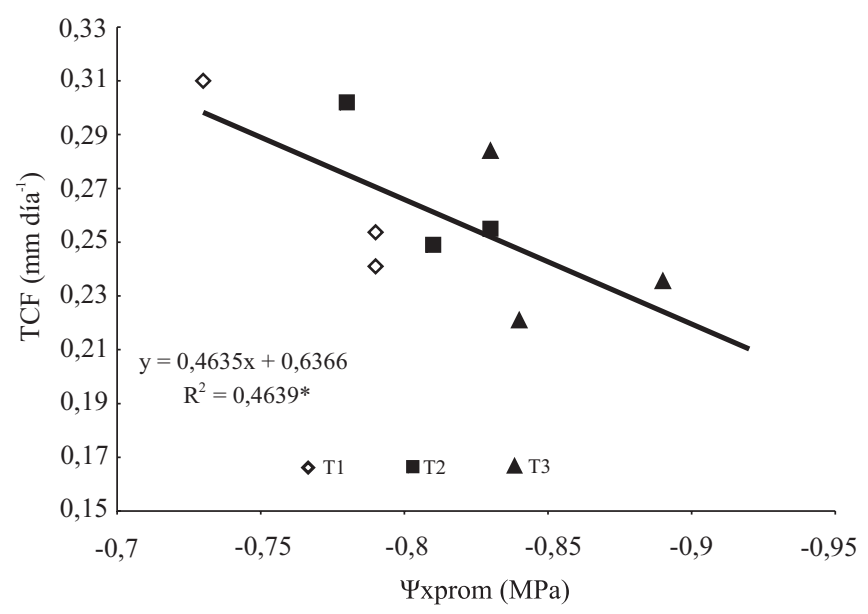

Figura 4. Relación entre la tasa de crecimiento de la baya (TCF),

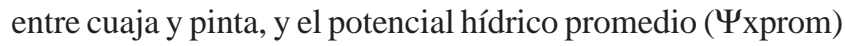
para el mismo período, para las tres temporadas. $\mathrm{T} 1$ : riego a $100 \%$ de la Etc durante toda la temporada; T2: riego con $75 \%$ de la Etc durante toda la temporada; T3: riego con 50\% de la Etc durante toda la temporada. medio de 21,1 $\mathrm{Mg} \mathrm{ha}^{-1}$; en el tratamiento T2, donde se aplicó en promedio el $67,6 \%$ de la Etc, se obtuvo un rendimiento medio de 21,1 $\mathrm{Mg} \mathrm{ha}^{-1}$; con aplicaciones de $38,3 \%$ de la Etc, se obtuvieron $17,7 \mathrm{Mg} \mathrm{ha}^{-1}$, y en el tratamiento T4 se obtuvieron, en promedio, 11,9 $\mathrm{Mg} \mathrm{ha}^{-1}$ exportables.

Durante el primer año, no existieron diferencias significativas entre tratamientos. En la segunda temporada, las diferencias se produjeron entre T1 y T2, con T3 y T4, siendo T4 el que presentó la menor producción. En la última temporada, existieron diferencias entre T1 y T2, con T4. T3 presentó una menor producción, pero la diferencia no fue significativa. Llama la atención la baja producción de T4 en cuanto a fruta exportable. Esto,

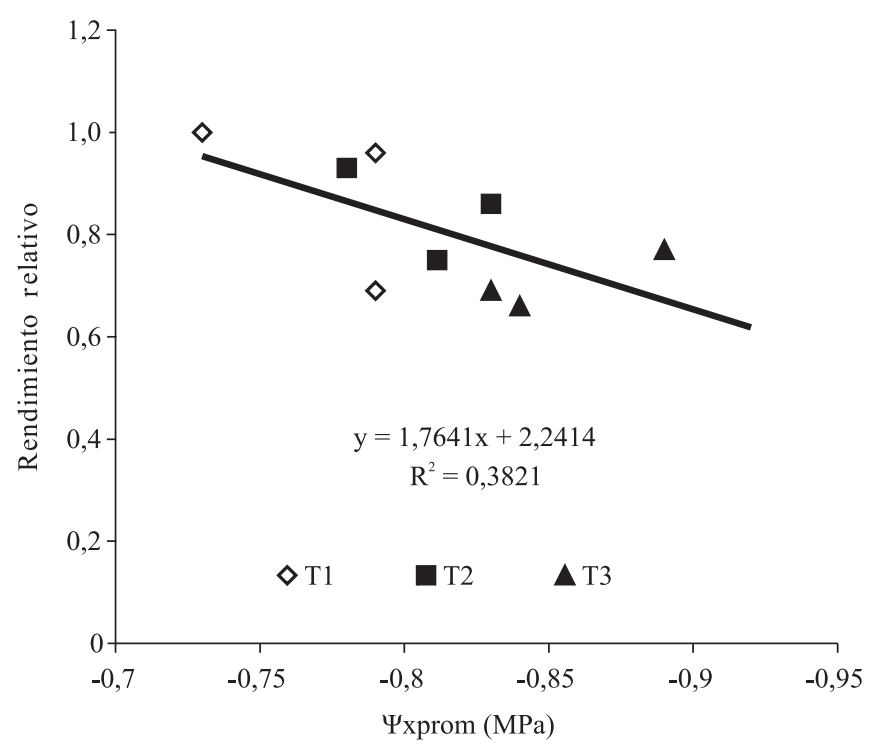

Figura 5. Relación entre la producción relativa, respecto del tratamiento T1, en el año de mayor producción, y el potencial

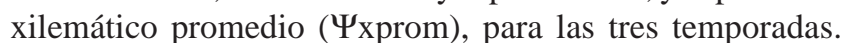
T1: riego a $100 \%$ de la Etc durante toda la temporada; T2: riego con $75 \%$ de la Etc durante toda la temporada; T3: riego con $50 \%$ de la Etc durante toda la temporada.

Cuadro 2. Producción de fruta exportable $\left(\mathrm{Mg} \mathrm{ha}^{-1}\right)$ para cada temporada, según tratamientos de riego ${ }^{(1)}$.

\begin{tabular}{lccc}
\hline Tratamiento & $2002 / 2003$ & $2003 / 2004$ & $2004 / 2005$ \\
\hline T1 & $17,14 \mathrm{a}$ & $24,14 \mathrm{a}$ & $25,02 \mathrm{a}$ \\
T2 & $18,77 \mathrm{a}$ & $21,44 \mathrm{ab}$ & $23,29 \mathrm{a}$ \\
T3 & $16,40 \mathrm{a}$ & $19,35 \mathrm{bc}$ & $17,35 \mathrm{ab}$ \\
T4 & $10,66 \mathrm{a}$ & $16,21 \mathrm{c}$ & $8,83 \mathrm{~b}$ \\
\hline
\end{tabular}

${ }^{(1)}$ Las letras iguales, en cada columna, indican que no existen diferencias significativas entre los tratamientos, según test de DSM, al 5\% de probabilidad. 
de alguna manera, podría estar asociado a un efecto acumulativo de los diferentes años de restricción de riego.

De acuerdo a los valores presentados en el Cuadro 2, existe, sin embargo, una tendencia clara entre la cantidad de fruta exportable y los tratamientos de riego, es decir, con el volumen de agua aplicado. Los valores de rendimiento exportable (Cuadro 2) se expresaron en términos relativos, respecto del tratamiento T1, para la temporada que presentó el valor más alto de producción, y se estableció una relación con las cantidades netas de agua aplicada en cada temporada, configurándose una función de producción relativa, la cual se presenta en la Figura 6.

Las funciones de producción agua-rendimiento se han realizado principalmente en cultivos anuales, y muy pocas en frutales y vides (Grimes \& Williams, 1990). Solamente se encontraron dos estudios anteriores de función de producción agua-rendimiento en vides, en variedades Waltham Cross y Thompson Seedless (Van Rooyen et al., 1980, Grimes \& Williams, 1990), en sistemas de conducción con porcentajes de cubrimiento menores al observado en parron español, utilizado en este estudio, el cual alcanza un porcentaje de sombreamiento del 85\% (Figura 7).
Debido a que las vides crecen en zonas con diferentes demandas evaporativas, la información se presentó

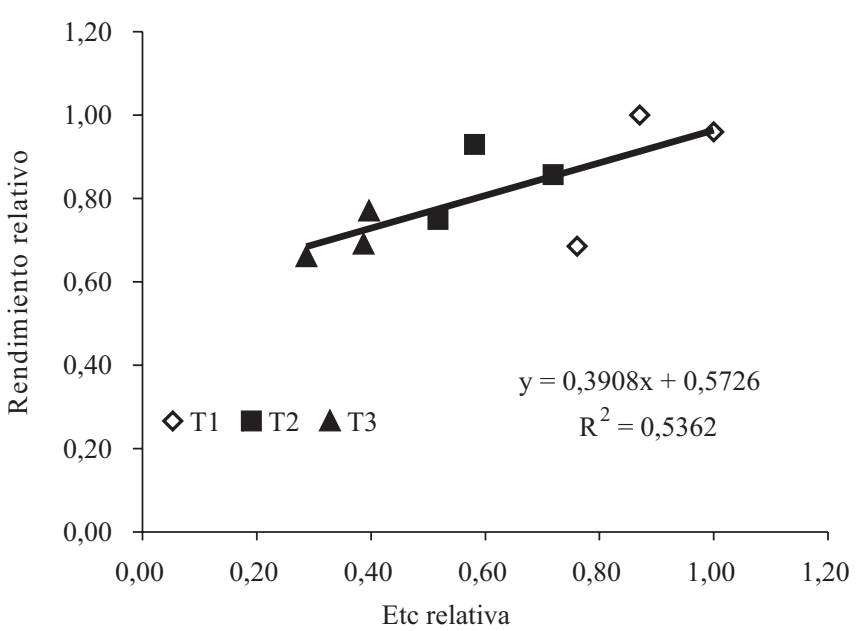

Figura 6. Relación entre la producción relativa y la evapotranspiración relativa del cultivo (Etc relativa), respecto del tratamiento T1, en el año de mayor producción. T1: riego a $100 \%$ de la Etc durante toda la temporada; T2: riego con $75 \%$ de la Etc durante toda la temporada; T3: riego con 50\% de la Etc durante toda la temporada.

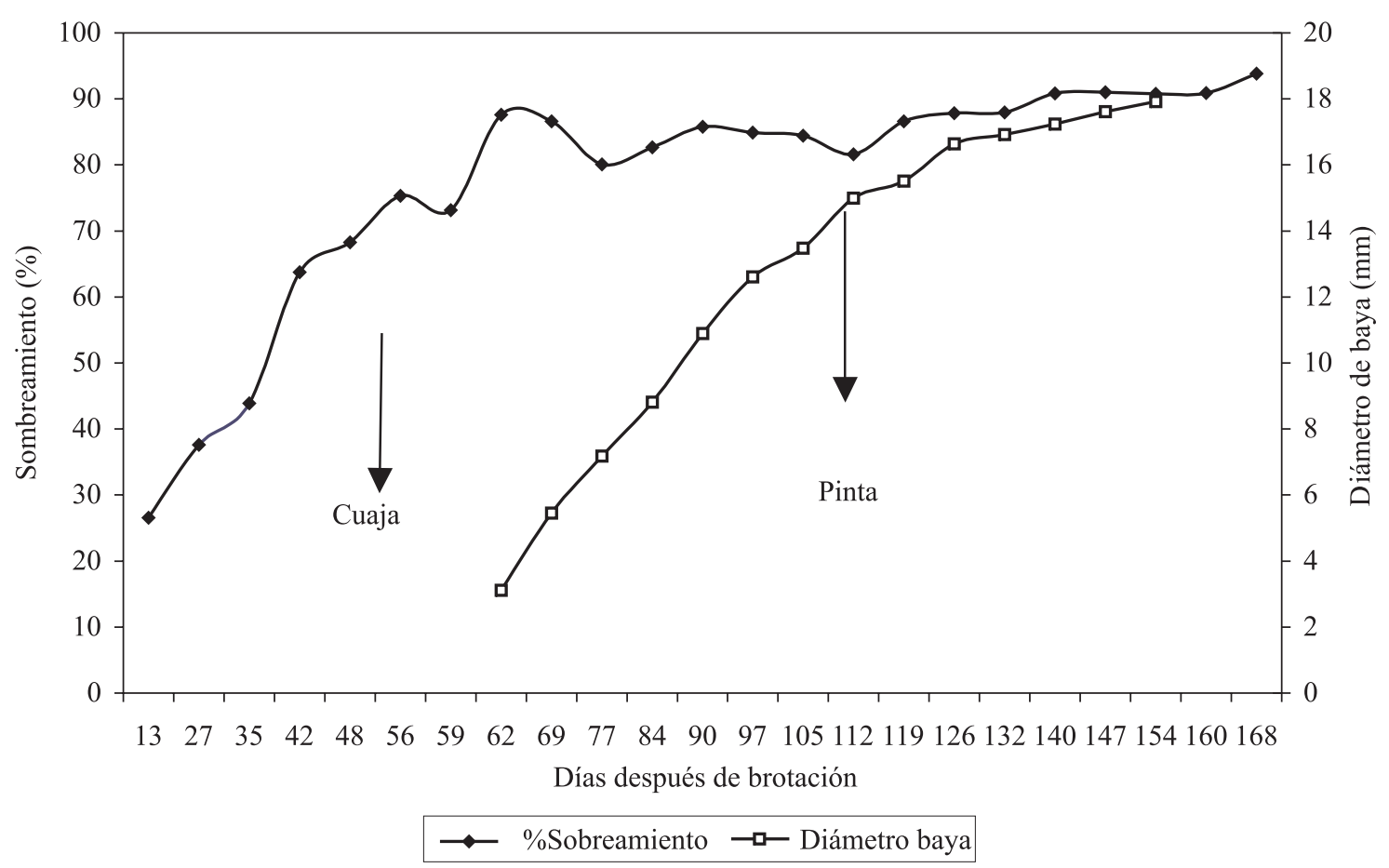

Figura 7. Evolución del porcentaje de sombreamiento, determinado a partir del índice de área foliar y diámetro de baya, para los diferentes tratamientos de riego, durante la temporada 2003/2004. 
en términos relativos (Figura 6), a fin de facilitar su uso. La máxima cantidad de agua aplicada, en este estudio (Quadro 1), fue de $1.056 \mathrm{~mm}$ en la temporada 2003/2004, y el valor más bajo fue de $303 \mathrm{~mm}$, en la temporada 2002/2003. En los estudios de funciones de producción agua-rendimiento, realizados por Van Rooyen et al. (1980) y Grimes \& Williams (1990), la cantidad máxima de agua aplicada fue de 230 y $790 \mathrm{~mm}$, respectivamente. Grimes \& Williams (1990), indican que al disminuir el agua en $60 \%$, el rendimiento disminuye en $35 \%$.

El rendimiento relativo se relacionó en forma lineal con la Etc relativa $\left(\mathrm{R}^{2}=0,54\right)$. En esta relación no se incluyó el T4, ya que este tratamiento consistió en aplicar estrés en periodos puntuales. En términos generales, la producción disminuye alrededor de $22 \%$ al disminuir el agua aplicada en alrededor de un 60\% (T3).

\section{Conclusiones}

1. La reducción de la disponibilidad de agua del suelo provoca una disminución del potencial hídrico xilemático medido a mediodía ( $\Psi$ xmin), por lo cual se puede utilizarlo como un indicador con fines de programación de riego.

2. Existe una relación entre el $\Psi x m i n$ y la tasa de crecimiento de las bayas, en el periodo fonológico de cuaja a pinta, siendo esa tasa mayor cuando el $\Psi$ xmin es superior a -0,75 MPa; este valor $\Psi$ xmin puede ser considerado como valor umbral para decidirse sobre la aplicación de riego.

3. La producción de fruta exportable en 'Crimson Seedless', cultivada en sistema de patronal español, disminuye cerca de un $22 \%$ al reducirse el agua aplicada en cerca de $60 \%$.

\section{Agradecimientos}

A Fondecyt - Chile, por el soporte financiero; a Agrícola Cabrini Hnos, quién facilitó el predio para realizar esta investigación; a la Sra. Cristina Aspillaga N., por su gran apoyo en las mediciones de terreno.

\section{Referencias}

CASSEL, D.K.; NIELSEN, D.R. Field capacity and available water capacity. In: KLUTE, A. (Ed.). Methods of soil analysis: physical and mineralogical methods. Madison: American Society of Agronomy; Soil Science Society of America, 1986. p.901-924.

CASTRO, M. Efectos de diferentes regímenes de riego sobre algunas relaciones agua/planta en vides (Vitis vinifera $\mathbf{L}$.) variedad Sultanina. 1997. 66p. Tesis (Licenciatura) - Universidad de Chile, Santiago.

CHONE, X.; LEEUWEN, C. van; DUBOURDIEU, D.; GAUDILLERE, J.P. Stem water potential is a sensitive indicator of grapevine water status. Annals of Botany, v.87, p.477-483, 2001.

DE LORENZI, F.; RANA, G. Sap flow transpiration in table grape vineyard growing in Southern Italy. Acta Horticulturae, v.537, p.6975, 2000.

FELMER, S.; ORTIZ, A. Respuesta de la vid (Vitis vinifera) cv. Sultanina a diferentes regímenes de agua y fertilización. 1997. 84p. Tesis (Licenciatura) - Universidad Mayor, Santiago.

FERERES, E.; GOLDHAMER, D. Deciduous fruit and nut trees. In: STEWARD, B.A.; NIELSEN, D.R. (Ed.). Irrigation of agricultural crops. Madison: American Society of Agronomy, 1990. p.987-1017. (Monograph, 30).

FERREYRA, R.; SELLÉS, G.; LEMUS, G. Efecto del estrés hídrico durante la fase II del crecimiento del fruto del duraznero cv. Kakamas en el rendimiento y estado hídrico de las plantas. Agricultura Técnica, v.62, p.565-573, 2002.

FERREYRA, R.; SELLÉS, G.; PERALTA, J.; VALENZUELA, J. Effect of water stress applied at different development periods of Cabernet Sauvignon grapevine on production and wine quality. Acta Horticulturae, v.646, p.27-33, 2004.

GRIMES, D.W.; WILLIAMS, L.E. Irrigation effects on plant water relations and productivity of Thompson Seedless grapevines. Crop Science, v.30, p.255-260, 1990.

HABU-HILAL, H.; COLLESANO, G.; WILLIAMS, L.E. The effect of rootstock and applied water on vine water status and gas exchange of Cabernet Sauvignon. Acta Horticulturae, v.526, p.163-167, 2000.

INTRIGLIOLO, D.S.; CASTEL, J.R. Feasibility of using LVDT and water mark sensor for irrigation scheduling in plums. Acta Horticulturae, v.664, p.317-323, 2004.

JONES, H.G. Irrigation scheduling: advantages and pitfalls of plantbased methods. Journal of Experimental Botany, v.55, p.24272436, 2004.

KOZLOWSKI, T. Responses of woody plants to flooding and salinity. Tree Physiology Monograph, v.1, p.1-29, 1997.

MATEO, R. Respuesta de la vid cv. Sultanina a diferentes regímenes de riego y fertilización. 2003. 93p. Tesis (Licenciatura) - Universidad de Chile, Santiago.

MEYER, W.S.; REICOSKY, D.C. Enclosing leaves for water potential measurement and its effect on interpreting soil induced water stress. Agricultural and Forest Meteorology, v.35, p.187-192, 1985.

NAOR, A. Irrigation and crop load influence fruit size and water relations in field-grown "Spadona" pear. Journal of the American Society for Horticultural Science, v.126, p.252-255, 2001.

NAOR, A. Relationships between leaf and stem water potential and stomatal conductance in three field-grown woody species. Journal of Horticultural Science and Biotechnology, v.73, p.431-436, 1998.

NAOR, A.; KLEIN, I.; DORON, I. Stem water potential and apple size. Journal of the American Society for Horticultural Science, v.120, p.577-582, 1995. 
PADGETT-JOHNSON, M.; WILLIAMS, L.E.; WALKER, M.A. Vine water relations, gas exchange, and vegetative growth on seventeen Vitis species grown under irrigated an non irrigated conditions in California. Journal of the American Society for Horticultural Science, v.128, p.269-276, 2003.

RICHARDS, D. The grape root system. Horticultural Reviews, v.5, p.127-168, 1983.

SCHOLANDER, P.F.; HAMMEL, H.T.; BRADSTREET, E.D.; HEMMINGSEN, E.A. Sap pressure in vascular plants. Science, v.148, p.339-346, 1965.

SHACKEL, K.A.; AHMADI, H.; BIASI, W.; BUCHNER, R.; GOLDHAMER, D.; GURUSINGHE, S.; HASEY, J.; KESTER, D.; KRUEGER, B.; LAMPINEN, B.; McGOURTY, G.; MICKE, W.; MITCHAM, E.; OLSON, B.; PELLETRAU, K.; PHILIPS, H.; RAMOS, D.; SCHWANKL, L.; SIBBETT, S.; SNYDER, R.; SOUTHWICK, S.; STEVENSON, M.; THORPE, M.; WEINBAUM, S.; YEAGER, J. Plant water status as an index of irrigation need in deciduous fruit trees. HortTechnology, v.7, p.23-29, 1997.
SELLES, G.; FERREYRA, R.; CONTRERAS, G.; AHUMADA, R.; VALENZUELA, J.; BRAVO, R. Manejo de riego en uva de mesa cv. Thompson Seedless, cultivada en suelos de textura fina. Agricultura Técnica, v.63, p.180-192, 2003.

SUCKEL, F. Calibración de métodos no destructivos de estimación de área foliar en vid, variedad Thompson Seedless. 2001. 56p. Tesis (Licenciatura) - Universidad de Chile, Santiago.

VAN ROOYEN, F.C.; WEBER. H.W.; LEVIN. I. The response of grapes to a manipulation of the soil-plant-atmosphere continuum. II. Plant-water relationships. Agrochemophysica, v.12, p.69-74, 1980.

VAN ZYL, J.L. Response of Colombar grapevines to irrigation as regards quality aspects and growth. South African Journal of Enology and Viticulture, v.5, p.19-28, 1984.

WILLIAMS, L.E.; PHENE, C.J.; GRIMES, D.W.; TROUT, T.J. Water use of mature Thompson Seedless grapevines in California. Irrigation Science, v.22, p.11-18, 2003. 